\title{
A study of joint damping in metal plates
}

\author{
Scott J.I. Walker, Guglielmo S. Aglietti, Paul Cunningham
}

University of Southampton, Southampton, UK

Received 22 December 2006; accepted 13 January 2009

\begin{abstract}
For satellite applications the determination of the correct dynamic behaviour and in particular the structural damping is important to assess the vibration environment for the spacecraft subsystems and ultimately their capability to withstand the launch vibration environment. Therefore, the object of this investigation is to experimentally analyse a range of aluminium panel configurations to study the effect of joints on the damping of the complete structure. The paper begins with a full description of the experimental method used to accurately determine the modal loss factors for each of the panel configurations analysed. Nine different panels were used in the experimental tests, six of which incorporate lap joints variations. The joint parameters investigated include fastener type, bolt torque, fastener spacing, overlap distance and the effect of stiffeners. The damping results of ten different joint variants are presented for each of the first twelve modes of vibration. This data is directly compared to the damping factors of an equivalent monolithic panel. Various specific conclusions are made with respect to each of the joint parameters investigated. However, the primary conclusion is that the mode shape combined with the joint stiffness and joint location can be suggestive as to the likely magnitude increase of the modal loss factor.
\end{abstract}

(C) 2009 Elsevier Ltd. All rights reserved.

\section{Introduction}

In the world of spacecraft engineering, the structural response to dynamic loads is crucial to assess its capability to withstand the launch environment. The dynamics and sound transmission characteristics of structures are determined by essentially three parameters: mass, stiffness and damping [1]. Damping is responsible for the eventual decay of the free vibrations in any system and as such it is an important parameter to determine when attempting to predict the dynamic behaviour of a structure. For satellite applications the determination of the correct dynamic behaviour and in particular the structural damping is important to assess the vibration environment for the spacecraft subsystems and ultimately their capability to withstand the launch vibration

E-mail address: sjiw@soton.ac.uk environment. To this day damping is still the dynamic characteristic that is least understood and the most difficult to quantify. The prediction of damping is particularly challenging for built up structures due to the limited knowledge of how joints affect the damping of the complete structure. It is therefore necessary to experimentally analyse increasingly complex structural joints to identify how specific joint parameters can affect the damping of the structure.

\section{Background}

To date little is known about the many detailed damping mechanisms inherent in structural joints and it remains an area of considerable research [2-4]. However, the two main mechanisms that are commonly referred to are air-pumping and friction. Various work has been performed to study these two mechanisms $[5,6]$ but the knowledge is not extensive enough to be generally 
applied with any accuracy. The core of the problem is the large quantity of possible variables that can define a joint in a structure. This investigation focuses on the experimental analysis of joint damping for aerospace application and as such, attempts to limit the possible number of joint variables to identify specific trends. The joint variables to be studied are:

- fastener type using bolts and rivets

- the effect of bolt torque

- the effect of fastener spacing

- the effect of overlap and separation distance between each line of fasteners

- the effect of an added stiffener

- the effect of two added stiffeners for larger plates

\section{Experimental approach}

\subsection{Test Specimens}

The test panels were cut and constructed from large sheets of 6082-T6 Aluminium with a thickness of $2 \mathrm{~mm}\left(E=70 \times 10^{9} \mathrm{~N} \mathrm{~m}^{-2}, \rho=2700 \mathrm{~kg} \mathrm{~m}^{-3}\right)$. The panels were sized to be large enough to be representative but small enough to be manageable for testing. It was also desirable to avoid square panels, reducing the likelihood of unusual symmetrical modes. Therefore the ratio of length to height was chosen to be around 0.7. A total of nine panel configurations were tested and constructed (denoted $A-I$ ). The starting point parameters for the panels are shown in Fig. 1.

Initially a monolithic panel along with two jointed panels were constructed to allow the effect of two different fasteners to be investigated, namely bolts and rivets. The location and spacing of the fasteners were identical for both jointed panel configurations forming two lines with a separation distance of $30 \mathrm{~mm}$. Seventeen
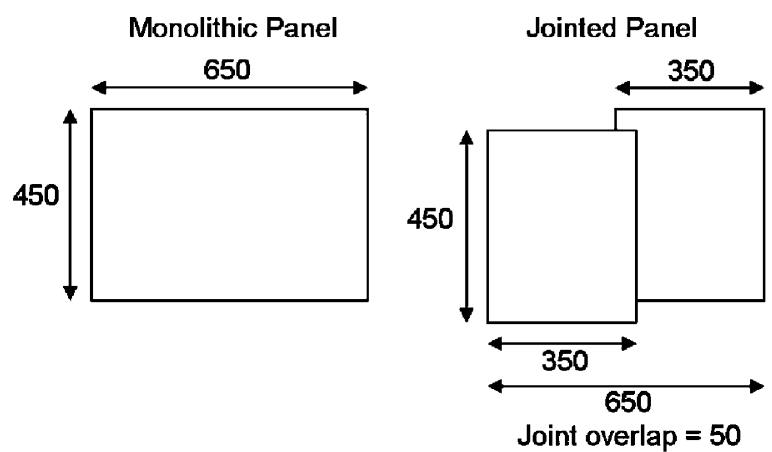

Fig. 1. Initial panel layout (not to scale, all dimensions in $\mathrm{mm}$ ).

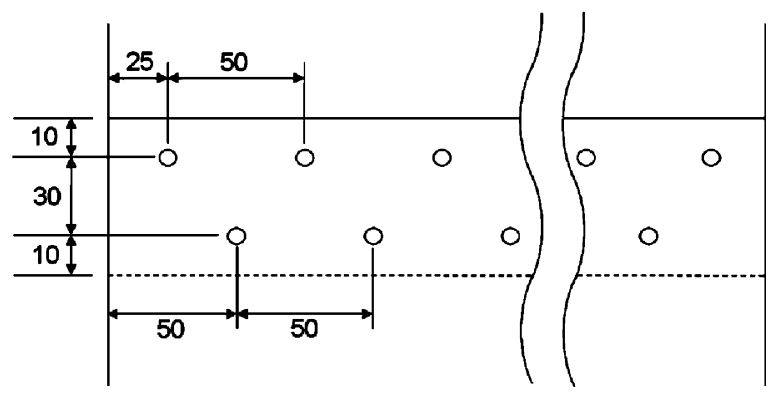

Fig. 2. Joint parameters (not to scale, all dimensions in $\mathrm{mm}$ ).

fasteners were used in each configuration and were staggered as shown in Fig. 2. The fasteners used were M5 bolts and $1 / 8^{\prime \prime}$ aircraft grade, countersunk rivets.

The three panel configurations were denoted as A, B and $\mathrm{C}$ for the monolithic, bolted and riveted panels, respectively. Six further panels were constructed to assess the remaining parameters listed in the previous section. Each configuration modified a single joint variable allowing direct data comparisons. A summary of all the panel configurations is shown in Table 1 . The general specifics of the joint locations for the larger panel (configuration I) are shown in Fig. 3.

\subsection{Test procedure}

Preliminary experimental tests were performed to determine the loss factors for the first eight modes of each configuration (up to around $110 \mathrm{~Hz}$ ), enabling a loss factor comparison based on the mode shape. The first eight modes shapes are displayed in Figs. 4 and 5. For these tests the panels were suspended from two mounting points and excited using a pendulum mounted impact hammer (as shown in Fig. 6(a)), allowing a high accuracy in the position of the excitation and repeatability. The resultant accelerations were measured using miniature tear drop shaped accelerometers (mass of $0.6 \mathrm{~g}$ ), displayed in Fig. 6(b). The signals from the accelerometers were captured on a computer at a sample rate of $5000 \mathrm{~Hz}$. Each vibration response was analysed using a sonogram [7], plotting time against frequency, enabling the decay of each mode to be linearly interpolated. The decay results for each mode were then averaged, allowing the standard deviation of the data to be determined. The linearity of the damping was quantified using RSQ values, which is the Square of the Pearson product moment correlation coefficient [8]. The closer the value is to 1 the more linear the result. Specific RSQ averages are stated for each of the test results presented in the following section. 
Table 1

Summary of test configurations A-I

\begin{tabular}{|c|c|c|c|}
\hline Configuration & General description & $\begin{array}{l}\text { Overall panel } \\
\text { dimensions }(\mathrm{mm})\end{array}$ & Joint specifics \\
\hline A & Monolithic panel & $650 \times 450$ & $\mathrm{n} / \mathrm{a}$ \\
\hline B & Single bolted joint & $650 \times 450$ & 17 fasteners ( $50 \mathrm{~mm}$ separation) in two lines $30 \mathrm{~mm}$ apart, $50 \mathrm{~mm}$ overlap \\
\hline $\mathrm{C}$ & Single riveted joint & $650 \times 450$ & 17 fasteners ( $50 \mathrm{~mm}$ separation) in two lines $30 \mathrm{~mm}$ apart, $50 \mathrm{~mm}$ overlap \\
\hline $\mathrm{D}$ & Single riveted joint & $650 \times 450$ & 43 fasteners ( $20 \mathrm{~mm}$ separation) in two lines $30 \mathrm{~mm}$ apart, $50 \mathrm{~mm}$ overlap \\
\hline $\mathrm{E}$ & Single riveted joint & $650 \times 450$ & 43 fasteners ( $20 \mathrm{~mm}$ separation) in two lines $20 \mathrm{~mm}$ apart, $40 \mathrm{~mm}$ overlap \\
\hline $\mathrm{F}$ & Single riveted joint & $650 \times 450$ & 43 fasteners ( $20 \mathrm{~mm}$ separation) in two lines $40 \mathrm{~mm}$ apart, $60 \mathrm{~mm}$ overlap \\
\hline G & Single riveted stiffener & $650 \times 450$ & 43 fasteners ( $20 \mathrm{~mm}$ separation) in two lines $30 \mathrm{~mm}$ apart, $50 \mathrm{~mm}$ overlap \\
\hline $\mathrm{H}$ & Large monolithic panel & $900 \times 600$ & $\mathrm{n} / \mathrm{a}$ \\
\hline I & Two riveted stiffeners & $900 \times 600$ & 59 fasteners ( $20 \mathrm{~mm}$ separation) in two lines $30 \mathrm{~mm}$ apart, $50 \mathrm{~mm}$ overlap \\
\hline
\end{tabular}

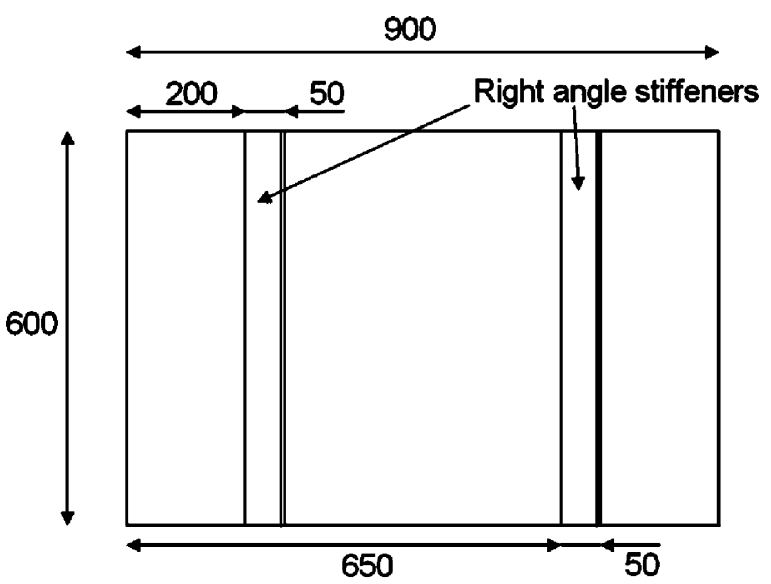

Fig. 3. Layout for configuration I (not to scale, all dimensions in $\mathrm{mm})$.

Initial tests were performed using both wax and glue mounted accelerometers and, as anticipated, it was found that for this frequency range the method of accelerometer mounting had no significant effect on the measured damping magnitude. Wax was therefore used throughout the testing campaign as the accelerometers had to be frequently moved between the mounting points.

\section{3. 'External influences' on the dynamics of the panel}

As the modal loss factors for metal plates (and especially aluminium plates) are known to be so low [1], any external influence on the panels vibration will noticeably affect the damping of the panel and therefore the accuracy of the data. For vibration testing using an impact hammer, the external damping influence primarily comes from two sources: the support mounting of the panel and any added mass/attachments on the panel, i.e. accelerometers.
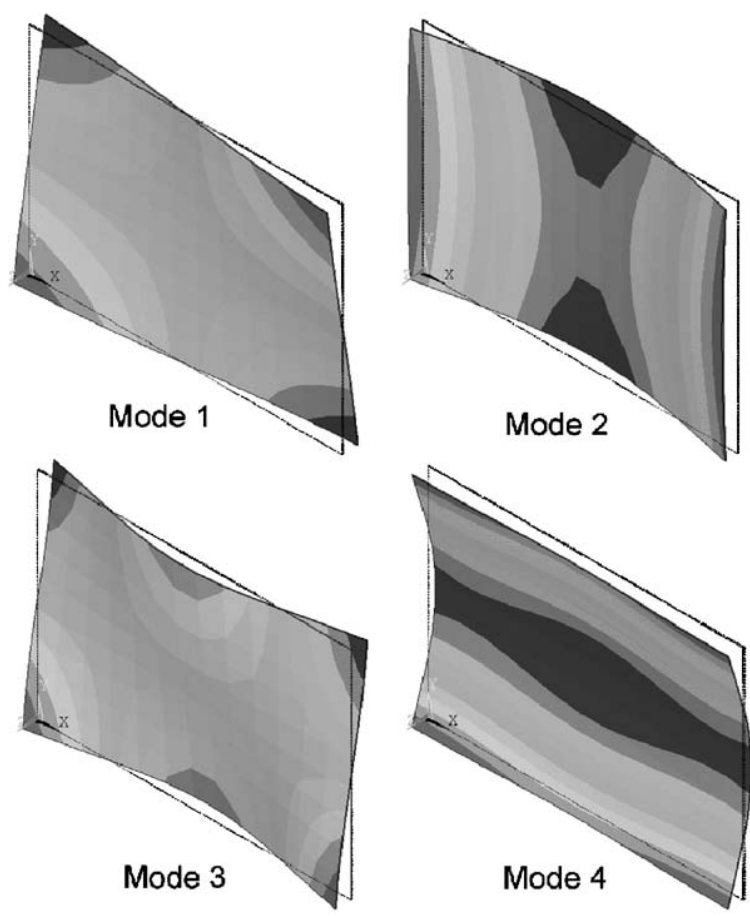

Fig. 4. General mode shapes 1-4.

In order to perform vibration tests on configurations A-C, the panels were suspended from two points located along the larger panel edge. However, it was found that, as expected, for certain mode shapes this resulted in a large loss factor variability as the mounting points created another source of damping of the panel. To overcome this problem the panels were mounted from the nodal points of each mode shape, minimising the excitation of the mounting locations. A direct comparison of the loss factor data for each mounting condition is shown in Fig. 7 for configuration A. The fixed and 
variable mounting tests have average RSQ values over the first eight modes of 0.940 and 0.995 , respectively.

It can be seen from the data that the loss factor variability (shown by the error bars measuring the standard deviation of the data) and magnitude increases for the mode shapes that excite the longer panel edge (i.e. modes shapes one and four). For the remaining mode

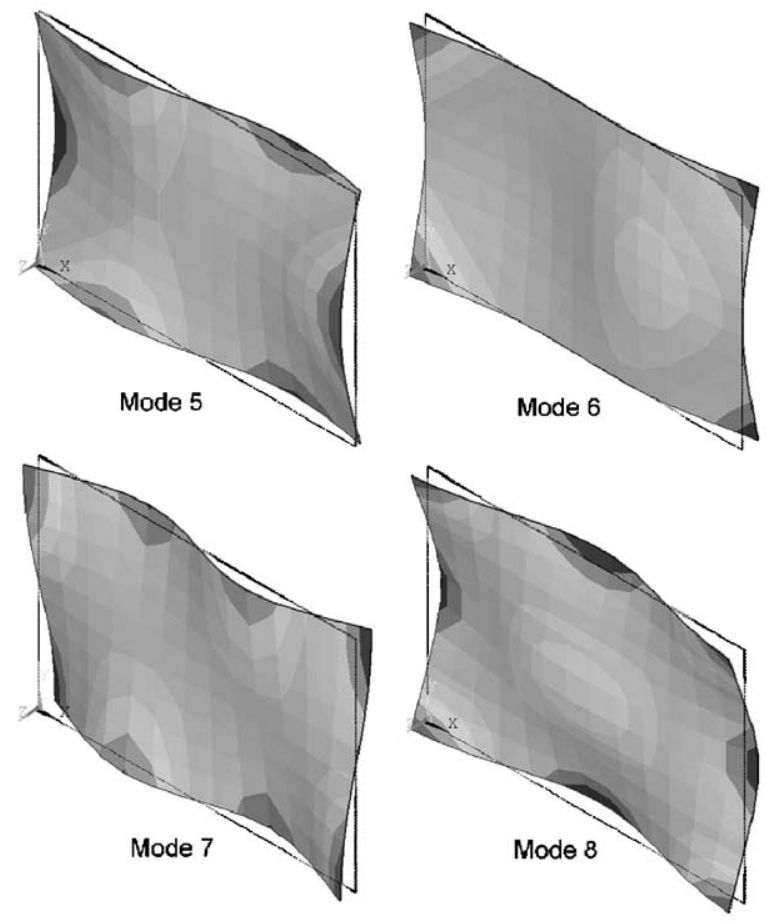

Fig. 5. General mode shapes 5-8. shapes the loss factor change is insignificant displaying the consistency of the data. As anticipated the variable mounting condition is the most accurate method for determining the loss factors, a trend seen not only from the graph but also the average RSQ values. However, the variable mounting test method is the most time consuming as every panel must be suspended using different mounting points for every mode and configuration. It is also limited to panels that enable the complete flexibility required to suspend the specimen from any location. For larger panels this becomes unfeasible (i.e. for configurations $\mathrm{H}$ and I). For these configurations partially variable mounting was used where the specimens were mounted from two points along the long edge of the panel. The location of these two points were optimised with respect to the nodal points of the mode shapes as much as possible but were not in the ideal positions for

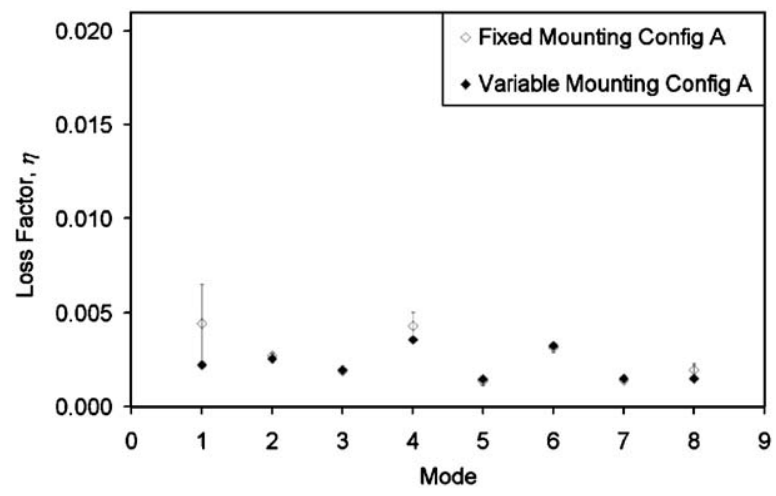

Fig. 7. Loss factors for fixed and variable mounting, configuration A.
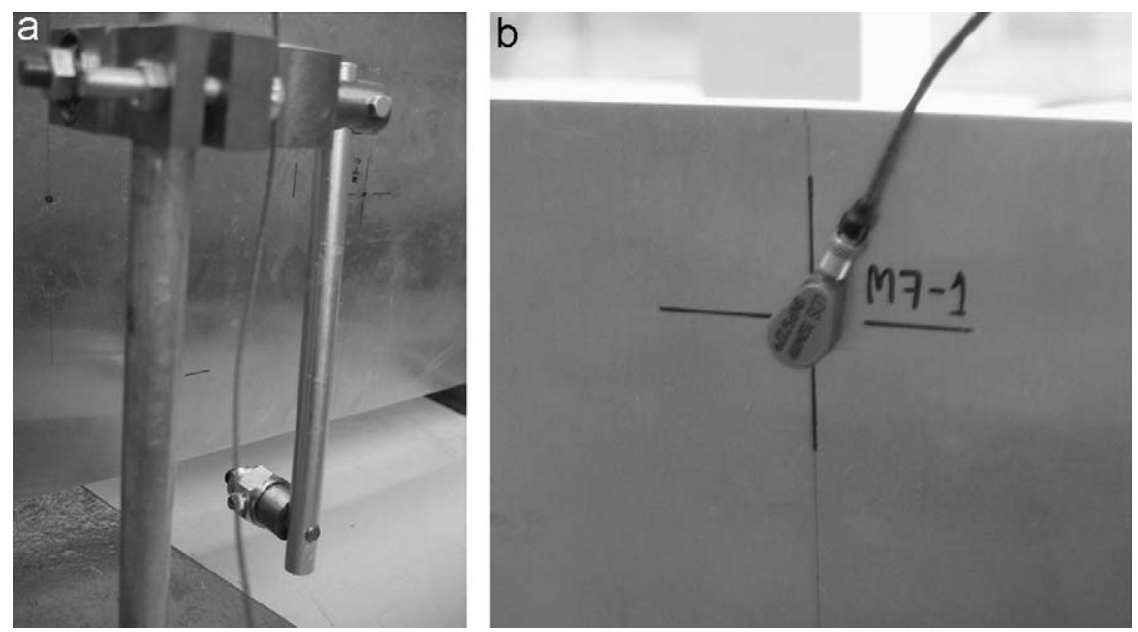

Fig. 6. Pendulum mounted impact hammer and tear drop accelerometer. 


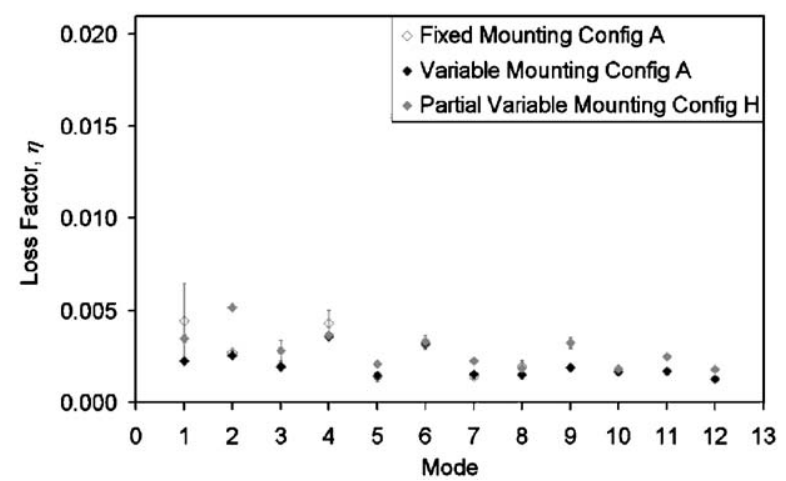

Fig. 8. Loss Factors for fixed and variable mounting, configurations $\mathrm{A}$ and $\mathrm{H}$.

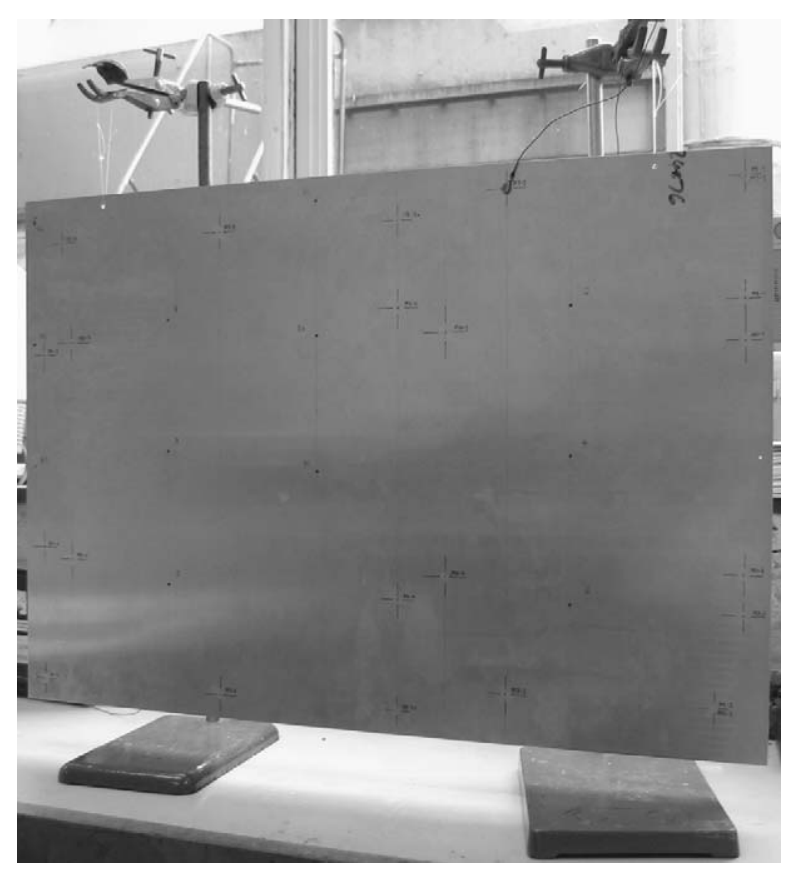

Fig. 9. Experimental setup for mode 7, configuration A.

all modes shapes. In total, four sets of mounting points were used for the larger panels. The loss factors for configuration $\mathrm{H}$ are shown in Fig. 8 alongside the previous data for configuration $\mathrm{A}$.

It can be seen from this figure that the loss factors for all the modes analysed have increased. This general rise in the damping is assumed to be due to the increase in the plate area, and subsequent increase of the damping effect due to the air around the panel. This can be most clearly seen for mode 2 which is the first longitudinal bending mode. The average RSQ value has also reduced to 0.925 over the first 12 modes, demonstrating an increase in the non linear damping behaviour. However, there has been no large increase in the standard deviation of the data when compared to the fixed mounting tests. It was therefore concluded that the partial variable mounting approach for the larger panels would produce data of sufficient accuracy for this investigation.

From the initial tests performed it was found that even the miniature accelerometers and connection cables had a noticeable influence on the damping magnitude when used in sufficient numbers. Four accelerometers were being used in the initial test case. It was therefore decided to use a single accelerometer to minimise this effect. Twenty five hammer hits were applied, per mode, per configuration and the resultant accelerations were measured at four antinode locations in turn using one accelerometer. The experimental setup for mode seven, configuration A is shown in Fig. 9.

\section{Experimental test results}

To investigate the effect of the type of fastener used and the bolt torque, configurations $\mathrm{A}-\mathrm{C}$ were experimentally analysed using the method described in the previous section. Configuration B was tested at three different torque magnitudes: $1.5,3$ and $4.5 \mathrm{Nm}$, resulting in five panel variants to be studied for the first three configurations. The average loss factors for these tests are displayed in Fig. 10.

It can initially be seen that the standard deviation of the results is quite low. This was a significant improvement over the preliminary tests due to the minimisation of the external influences on the damping of the panel [9]. The first major trend identified from this data is that the loss factors of certain mode shapes are very sensitive to a reduction in the joint stiffness. Modes 1 , 2, 7 and 9 clearly display this trend. It has been shown previously [9] that this is due to the specific displacements of the panel at the location of the joint. These modes shapes open up the joint, increasing the effect of gas pumping and therefore increasing the loss factors. These modes were defined as critical modes. The non critical modes do not try to force open the joint and this results in a very low loss factor variation as the stiffness of the joint changes. Typical examples of this are modes 3, 4 and 6. (The displacements for these modes are displayed in Figs. 4 and 5). It was the identification of the critical mode shapes that led to the increase in the number of modes under investigation. Modes 9 and 12 were theoretically identified to be critical and were therefore included in the experimental tests. The average RSQ values for configurations A, B $1.5 \mathrm{Nm}, \mathrm{B}$ 


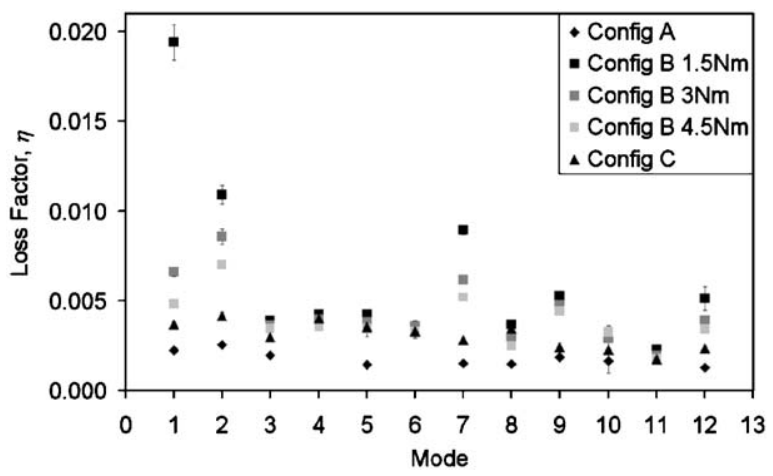

Fig. 10. Experimental loss factors for configurations A, B and C.

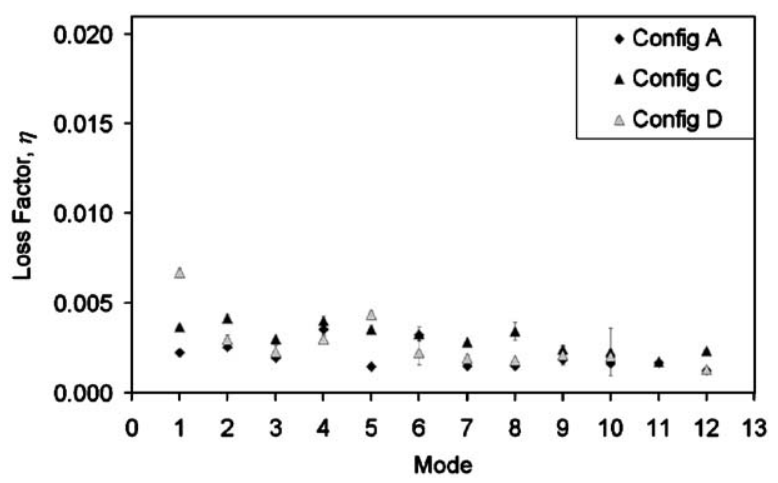

Fig. 11. Experimental loss factors for configurations A, C and D.

$3 \mathrm{Nm}, \mathrm{B} 4.5 \mathrm{Nm}$ and C were $0.994,0.974,0.971,0.969$ and 0.921 over the first 12 modes, respectively. These results show that, as expected, the most linear damping was seen for configuration A. A small loss in the linearity of the data was found for Configuration B. However the averaged RSQ values were consistent over the three tested configurations suggesting a small repeatable non linear trend. The lowest RSQ was seen for the riveted configuration suggesting a greater susceptibility for non linear damping decay.

For the general trends under investigation it was found that the riveted joint consistently resulted in lower loss factor magnitudes when compared to the bolted joint torqued to $4.5 \mathrm{Nm}$. It was also shown that as the bolt torque increased the loss factors reduced. The rate of this reduction was highly dependant on the mode shape (leading to the classification of critical and non critical modes [9]).

To determine the impact of fastener spacing on the damping of the panels, the data for configurations A and $\mathrm{C}$ were plotted alongside the data for configuration $\mathrm{D}$ as shown in Fig. 11.

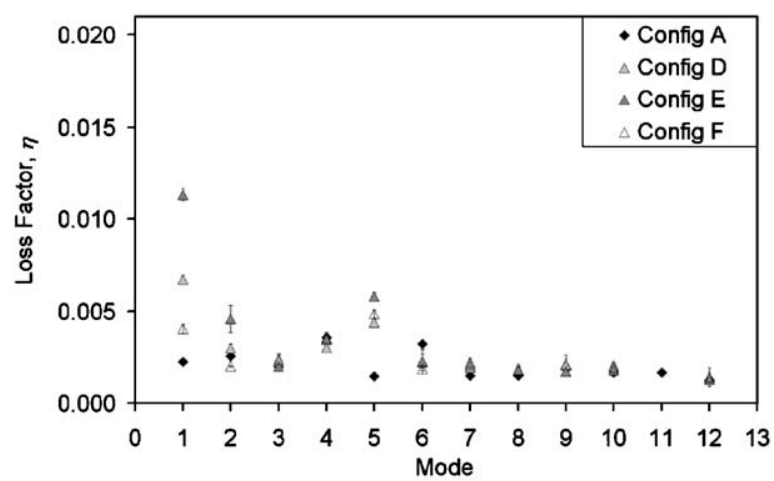

Fig. 12. Experimental loss factors for configurations A, D, E and F.

It can be seen that the general trend as a result of increasing the number of fasteners along the joint is the reduction of the loss factor magnitudes. However, modes 1 and 5 are the exceptions to this rule. The results also display a general trend of reducing loss factor magnitudes as the frequency increases, a trend which was also displayed by the non critical modes in Fig. 10. The largest magnitude increase is for mode 1, which could be ascribed to an increase in the friction along the joint. The average RSQ value for configuration D is 0.957 , which again is lower than the bolted configuration, implying a more non linear behaviour for riveted joints. A further conclusion from the data shown in Fig. 11 is that above a certain joint stiffness the joint classification becomes unnecessary as there is little to suggest any influence of the critical modes on the loss factor magnitudes. However, Fig. 10 implies that the bolt torque required to achieve the stiffness threshold would be greater than $4.5 \mathrm{Nm}$.

To study the overlap and separation distance between the lines of fasteners, two further configurations were experimentally analysed, the first with a smaller lap joint and the second with a larger lap joint (see Table 1 for further details). The results of these configurations are presented together with those of configurations A and D in Fig. 12.

The first observation that can be made from this data is that for these stiffer joints there is very little damping variation above mode 6 . Modes 7-12 all follow the same trends displayed by the monolithic panel, resulting in the conclusion that for stiff joints the damping is dominated by the first few mode shapes. It can be seen from modes 1 and 2 that the smaller the joint overlap the higher the loss factor magnitude. This suggests that despite the increased overlap area, the increased stiffness of the wider overlap and fastener line spacing has resulted in a reduction of the loss factors. The 


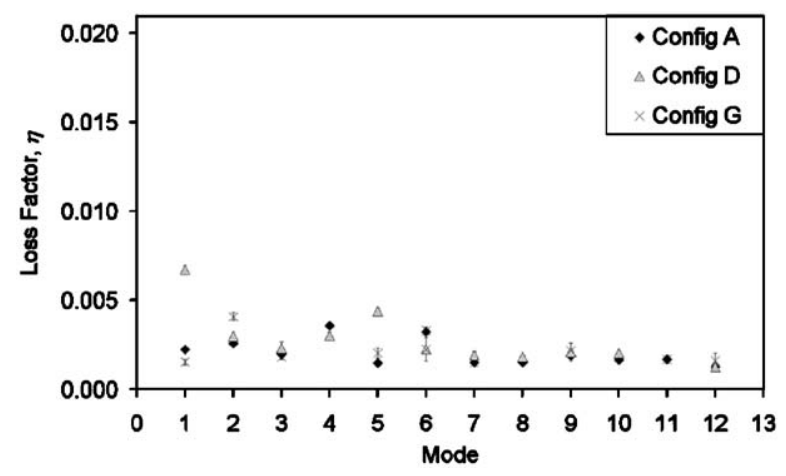

Fig. 13. Experimental loss factors for configurations A, D and G.

average RSQ values for configurations $\mathrm{E}$ and $\mathrm{F}$ are 0.954 and 0.937 , respectively, which is the same order of magnitude as the previous riveted joint data. The final comparison to be performed for the smaller panels was the attachment of a right angle stiffener instead of a lap joint. The specifics of the fasteners are equivalent to that of configuration $\mathrm{D}$, and is therefore plotted with this data and that of configuration A in Fig. 13.

Once again the addition of this stiff joint has had a negligible impact on the loss factor magnitudes of the higher modes. The damping of modes 7-12 again follow the same trends as the monolithic panel. However, it can also be seen that there is little significant change in the loss factor magnitudes for the low modes. The largest change is shown for mode 2, which is the first longitudinal mode of bending. The low frequency torsional modes remain largely unaffected by the addition of the stiffener as the vibration of main panel does not have to cross a joint connecting two plates. This significantly improves the transmitability of the vibration, and therefore displays damping magnitudes similar to that of the monolithic panel. This is reflected in the linearity of the data with an average RSQ value of 0.985 for configuration $\mathrm{G}$.

The final experimental tests analysed a larger panel with two riveted right angle stiffeners. As the panel size had increased, an equivalent larger monolithic panel was required to allow a direct comparison of the results. The monolithic panel and the equivalent stiffened panel are denoted by $\mathrm{H}$ and I, respectively (as outlined in Table 1). The results of these experimental tests are plotted with configurations $A$ and $G$ in Fig. 14. It can be seen from the data that again, for the higher frequency mode shapes, there is comparably little magnitude change in the loss factors. The most significant change is the magnitude increase for modes 1 and 2 which has increased to a level almost comparable to

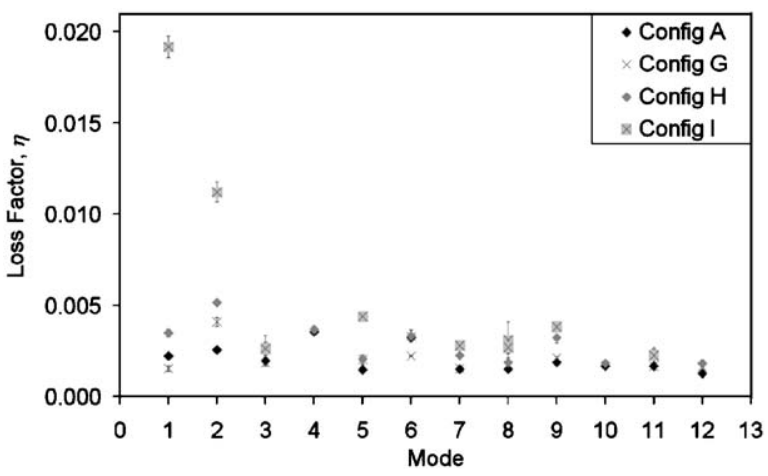

Fig. 14. Experimental loss factors for configurations A, G, H and I.

configuration B with $1.5 \mathrm{Nm}$ of torque. There are a number of possible reasons for this large rise. Firstly the jointed area has increased by a factor of more than 2.5 . Secondly the joint position relative to the mode shape has changed, influencing not only the effect of friction and gas pumping, but also the mass distribution of the panel. The averaged RSQ value for configuration I was found to be 0.941 which is equivalent to the riveted joints.

\section{Conclusions}

This publication has experimentally investigated how specific joint parameters affect the damping of metal plates for aerospace applications. As anticipated, the riveted joint, due to its higher stiffness, resulted in lower loss factors when compared to an equivalent bolted joint. It was also found that as the bolt torque reduced, the damping of the panel increased. However, the size of the increment was dependant on the specific displacements of the mode shapes, classified into 'critical' and 'non critical' modes. This trend was true for the weaker joints with fewer fasteners. When the stiffness of a joint increases above a threshold level, these mode shape classifications become unnecessary due to the dominance of the damping of the lower modes of vibration. It was found that the effect of a joint overlap is dependant the stiffness change of the joint. If the stiffness increases with the overlap then the damping reduces despite the increase in overlap area. An equivalent panel with an added stiffener suggested that if the stiffness of the whole panel was not dominated by the stiffness of a joint in the material then the damping remained close to that of an equivalent monolithic panel. This trend was not found to be repeated for a larger panel with two added stiffeners as the jointed area had increased and moved with respect to the mode shape. 
Average RSQ values have also been presented suggesting linearity trends for the various joint configurations. However, these values need to be further investigated with respect to specific mode shapes before any detailed conclusions can be made.

The main conclusion of this paper is the important effect that joint stiffness and location with respect to mode shape, have on the damping. For example, a low stiffness joint can result in high or low loss factors depending on where on the panel the joint is located. It is therefore necessary to study the joint location as the next variable to be investigated.

\section{References}

[1] E. Ungar, Noise and Vibration Control Engineering: Principles and Applications, Wiley, New York, 1992, ISBN 0-47161751-2.

[2] A.S. Phani, Damping Identification in Linear Vibration, Ph.D. Thesis, St. John's College, Cambridge, January, 2004.
[3] A. Salzmann, S. Fragomeni, Y. Loo, The damping analysis of concrete beams under free-vibration, Advances in Structural Engineering 6 (1) (2003) 53-64.

[4] R.F. Gibson, Non traditional application of damping measurements, in: Symposium on M3D: Mechanics and Mechanisms of Material Damping, 1992, pp. 60-75, STP 1169.

[5] S. Earles, Theoretical estimation of the frictional energy dissipation in a simple lap joint, Journal of Mechanical Engineering Science 8 (2) (1966) 207-214.

[6] G. Maidanik, Energy dissipation associated with gas pumping in structural joints, Journal of Acoustic Society of America 40 (5) (1966) 1064-1072.

[7] C. Hodges, J. Power, J. Woodhouse, The use of the sonogram in structural acoustics and an application to the vibrations of cylindrical shells, Journal of Sound and Vibration 101 (2) (1985) 203-218.

[8] S. Hays, An Outline of Statistics, sixth ed., Longmans, New York, 1962.

[9] S. Walker, G. Aglietti, P. Cunningham, Experimental Analysis of Damping Across Joints in Metal Plates, in: Proceedings of the Sixth International Conference on Modern Practice in Stress and Vibration Analysis, 2006, Bath, UK. 